\title{
Dissecting the Nature of Exciton Interactions in Ethyne-linked Tetraarylporphyrin Arrays
}

\author{
Stefano Caprasecca, ${ }^{*, \dagger}$ Carles Curutchet, ${ }^{*, *}$ and Benedetta Mennucci, ${ }^{* \dagger}$
}

Dipartimento di Chimica e Chimica Industriale, University of Pisa, Via Risorgimento 35, 56126

Pisa, Italy, and Departament de Fisicoquímica, Facultat de Farmàcia, Universitat de Barcelona, Av. Joan XXIII s/n, 08028 Barcelona, Spain

E-mail: stefano.caprasecca@for.unipi.it; carles.curutchet@ub.edu; bene@dcci.unipi.it

\footnotetext{
${ }^{*}$ To whom correspondence should be addressed

†ipartimento di Chimica e Chimica Industriale, University of Pisa, Via Risorgimento 35, 56126 Pisa, Italy

\#Departament de Fisicoquímica, Facultat de Farmàcia, Universitat de Barcelona, Av. Joan XXIII s/n, 08028 Barcelona, Spain
} 


\begin{abstract}
We investigate how electronic energy transfer in a series of three ethyne-linked Zinc- and free base-tetraarylporphyrin dimers is tuned by the type of linker and by substitution on the porphyrin rings. We use TD-DFT combined with a recently developed QM/MM/PCM method allowing a mixed fully polarizable description of the chromophores and the bridge. This allows us to dissect the bridge-mediated contributions to energy transfer in terms of superexchange (through-bond) interactions and Coulomb (through space) terms mediated by the polarizability of the bridge. We explore the effects of the substituents and of the bridge-chromophore mutual orientation on these contributions. We find that bridge-mediated superexchange contributions largely boost energy transfer between the porphyrin units. When the effect of the solvent is also considered through PCM, we find good agreement with the through-bond versus throughspace contributions determined experimentally, thus indicating the need to properly include both solvent and bridge effects in the study of energy transfer in bridged molecular dyads.
\end{abstract}

\title{
1 Introduction
}

Multiporphyrin architectures have been largely investigated as potential efficient photonic devices; indeed they possess many attractive features: a rigid and planar geometry, a high stability, an intense electronic absorption and a small HOMO-LUMO gap; moreover, their optical and redox properties can be tuned by appropriate metallation. ${ }^{1-3}$ Different types of covalently linked porphyrin arrays with linear, cyclic, and cross-linked geometries have been constructed and used to achieve a thorough understanding of all factors that affect electronic communication among the various constituents. ${ }^{4,5}$ Actually, only through a detailed analysis of these factors and their coupling it is possible to develop a rational design of a wide range of molecular devices to be used for photonic applications.

An important part of the investigations reported so far on multiporphyrin architectures has been focussed on electronic energy transfer (EET) processes, which together with electron transfers determine the electronic communication. In these arrays a major issue concerns the extent 
of communication that is mediated by the linker. In covalently linked porphyrin arrays, indeed, a semirigid linker is generally introduced between the donor and acceptor chromophores (metal- and free base-porphyrins, respectively) in order to keep a well-defined and rigid structure and maintain some properties of the isolated chromophores, so that the resulting complex has predictable characteristics, while imparting efficient electronic communication channels among the chromophores. ${ }^{6,7}$ Among the linkers having these characteristics, ethyne linkage between aryl groups have been used in combination with different porphyrin constituents, because of their small attenuation factors. ${ }^{8}$ In particular, Lindsey and collaborators have conducted detailed spectroscopic investigations, both static and time-dependent, to rationalize the role of different factors in determining the EET efficiency, and more in general the electronic communication in complexes where the porphyrin constituents are linked via diphenylethyne groups at the meso positions (see Figure 1 for a representation of the complexes investigated). ${ }^{6,9}$
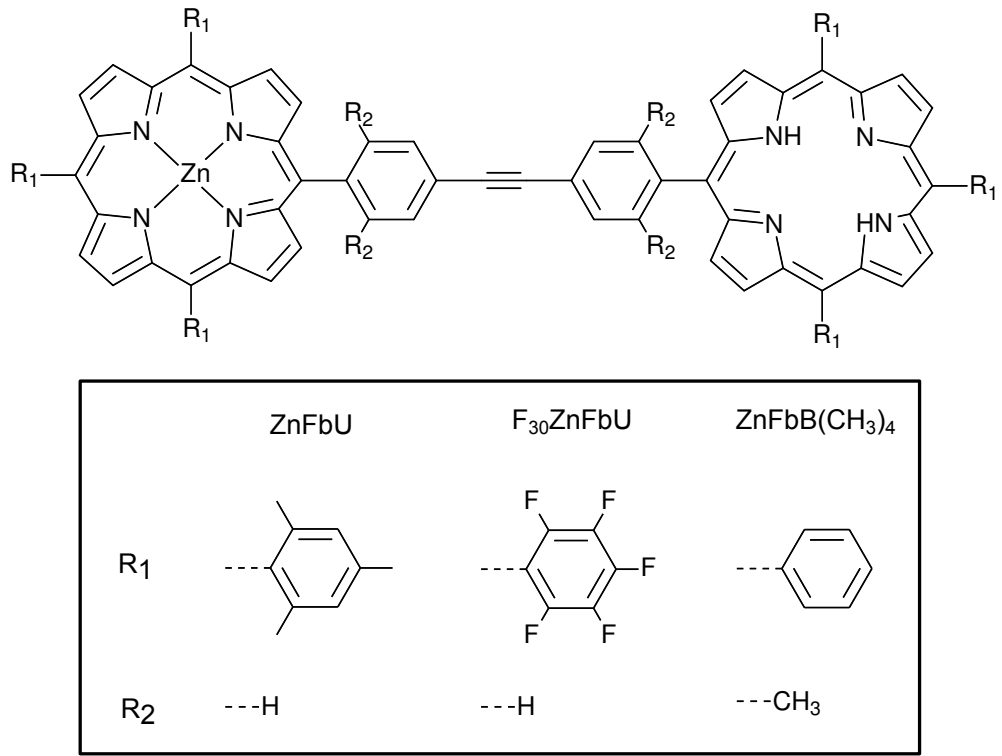

Figure 1: Schematic representation of the geometry of the three porphyrin dimers studied.

From their analysis on different Zinc- and free base-porphyrin dimers they concluded that energy transfer primarily proceeds via a process mediated by the diarylethyne linker but, remarkably, they also observed that the rate of such transfers can change by orders of magnitude if the chromophores are slightly modified, despite keeping the same linker. For example the energy transfer 
rates of two diphenylene ethynylene-linked bisporphyrin systems $\left(\mathrm{F}_{30} \mathrm{ZnFbU}\right.$ and $\mathrm{ZnFbU}$, see Figure 1) differ by a factor 10 despite the fact that they contain the same linker. As the energy transfer in $\mathrm{ZnFbU}$ is extremely rapid and essentially quantitative, the slower dynamics observed for $\mathrm{F}_{30} \mathrm{ZnFbU}$ was attributed to attenuated energy transfer and not to the onset of competing processes associated with the fluorination of the phenyl rings. The main issue is therefore to understand the mechanism beyond the different EET efficiencies due to minor changes in the chromophores and the real role played by the linker.

It is well known that the EET mechanism in these systems involves both through-space (TS) and through-bond (TB) contributions. The former is mediated by the standard Coulomb interaction between the two transition densities localized on the donor and acceptor moieties, while the TB contribution involves interactions beyond the coulombic terms and is generally explained in terms of D/A orbital overlap, i.e. an electronic exchange interaction. Historically the TS mechanism was modelled using the Förster dipole-dipole approximation ${ }^{10}$ (see Equation 3), while a Dexter-like picture was introduced for the TB one, where the EET rate constant presents an exponential decay with the D/A distance. ${ }^{11}$ Deviations from what is expected on the basis of the two idealized models have most frequently been ascribed to superexchange, ${ }^{12-14}$ a longer-range exchange mechanism allowed by the presence of intervening structures, such as linkers, between the donor and acceptor moieties. Another possible explanation of the deviations sometimes observed in bridge-mediated singlet-singlet EET rates is that the bridge polarizability can explicitly affect the Coulomb interaction between the D/A moieties. ${ }^{15-18}$ Very recently we have shown that this is indeed the case for the EET between the bright singlet states in the $\mathrm{ZnFbB}\left(\mathrm{CH}_{3}\right)_{4}$ complex. ${ }^{19}$ In that study we applied a fully-coupled quantum-mechanical/discrete/continuum method we recently developed to model energy transfer processes, which combines a continuum dielectric description of the environment (in its Polarizable Continum Model, PCM, formulation ${ }^{20}$ ) with a flexible definition of QM and polarizable $\mathrm{MM}\left(\mathrm{MMPol}^{21}\right)$ regions. This three-level method allowed us to describe bridge-mediated contributions by employing either a quantum mechanical or a polarizable MM description for the linker, combined with a continuum model for solvent effects. Such an approach is here applied to 
the full set of Zinc- and free base-porphyrin dimers studied by Lindsey and collaborators (ZnFbU, $\mathrm{F}_{30} \mathrm{ZnFbU}$ and $\left.\mathrm{ZnFbB}\left(\mathrm{CH}_{3}\right)_{4}\right)$, in order to dissect the contributions to the electronic communications. In particular, the aim is to quantify the TS vs. TB contribution, and also to give a detailed explanation of the role played by the linker and by different substituents on the D/A moieties in the final EET efficiency. Note that in the previous sentence, and in the rest of the paper from here, we will use a definition of TS and TB contributions consistent to that employed by Lindsay and collaborators, i.e., we will label TB the bridge-mediated contribution to the total coupling that is QM in character, mediated by orbital overlap and exchange, and TS the Coulomb contribution, which can be described including the bridge classical polarizability. Such definitions differ from the standard ones in that they both include the effects of the bridge.

\section{Methods and computational details}

The method we use to analyse the EET properties of the porphyrin dimers combines a continuum dielectric description of the solvent with either a fully quantum mechanical (QM) description of the porphyrin dimer, or alternatively a QM description of the photoactive moieties combined with a polarizable MM description of the linker.

The continuum solvent description is based on the IEF formulation of the PCM model, ${ }^{22}$ using the discretization from surface charge density into point charges available in Gaussian09 (requiring the Gaussian03 defaults). Within this framework, the QM system is embedded in a cavity of shape and dimension defined according to the geometrical structure of the solute, and thus also depending on the distance and relative orientation of the chromophores. The solvent is described as a polarizable continuum (characterized by its dielectric constant and refractive index), which responds to the presence of the QM system through a set of induced (or apparent) charges placed on the surface of the molecular cavity. In turn, such charges act back on the QM system from which they are generated: this mutual polarization effect is solved through a modified self-consistent field scheme. In addition, within the PCM framework it is possible to introduce nonequilibrium effects 
that arise whenever a fast process in the QM system originates delays in the response of the solvent. This is exactly what happens during an electronic excitation or during an EET, when the energy donor, $\mathrm{D}$, is de-excited by exciting the acceptor, A. In the common time-scales of these processes, the response of the solvent is incomplete in the sense that only its fast degrees of freedom (of electronic nature) can equilibrate with the final state of the QM system, while the rest remains frozen in the initial configuration corresponding to the QM system before the change.

On the other hand, the MMPol method adopts an atomistic description of the environment based on a classical polarizable force field based on the induce dipole model. ${ }^{21}$ In this context, the QM charge distribution polarizes the MM region, and the corresponding MM induced dipoles, as well as the MM point charges, then polarize back the QM part of the system. Thus, as in PCM, mutual polarization between the QM and MM regions is solved through a modified selfconsistent field scheme. In this work, we also use a combined QM/MM/PCM scheme we recently developed, ${ }^{19}$ in which full mutual polarization among the QM, MM and PCM regions is accounted for.

In all the calculations, the EET coupling $V$ is obtained using a linear response (LR) approach where vibronic effects are neglected. In the model we have developed, ${ }^{21,23,24}$ the transition densities of donor and acceptor $\left(\rho^{\mathrm{T}}\right)$ are used instead of dipolar or multipolar approximations; as a result, the coupling for the isolated D/A pair becomes:

$$
V_{0}=\int d r^{\prime} \int d r \rho_{\mathrm{D}}^{\mathrm{T} *}\left(r^{\prime}\right) \frac{1}{\left|r-r^{\prime}\right|} \rho_{\mathrm{A}}^{\mathrm{T}}(r)+\int d r^{\prime} \int d r \rho_{\mathrm{D}}^{\mathrm{T} *}\left(r^{\prime}\right) g_{\mathrm{xc}}\left(r^{\prime}, r\right) \rho_{\mathrm{A}}^{\mathrm{T}}(r)-\omega_{0} \int d r \rho_{\mathrm{D}}^{\mathrm{T} *}(r) \rho_{\mathrm{A}}^{\mathrm{T}}(r),
$$

where $g_{x c}$ is the exchange-correlation kernel determined by the specific functional used, whereas the last term is an overlap contribution weighted by the resonance transition energy $\omega_{0}$.

Within the combined MMPol/PCM framework, ${ }^{19}$ the polarization effect of the solvent and the linker on the electronic coupling is not introduced as a screening factor as in the Förster model but it enters in the definition of the coupling itself through an additional term (called explicit term) 
which sums to the $V_{0}$ defined in Equation 1. Particularly, if we mimic the solvent and linker polarization induced by the donor transition density in terms of PCM charges and MM induced dipoles, respectively, such an additional term becomes:

$$
V_{\text {solv }}=\sum_{t}\left[\int d r \rho_{\mathrm{A}}^{\mathrm{T}}(r) \frac{1}{\left|r-s_{t}\right|}\right] q_{t}\left(s_{t} ; \varepsilon(\omega), \rho_{\mathrm{D}}^{\mathrm{T}}\right)-\sum_{p}\left[\int d r \rho_{\mathrm{A}}^{\mathrm{T}}(r) \frac{r_{p}-r}{\left|r-r_{p}\right|^{3}}\right] \mu_{p}\left(r_{p} ; \rho_{\mathrm{D}}^{\mathrm{T}}\right),
$$

where the frequency-dependent dielectric permittivity $\varepsilon(\omega)$ reduces to the dynamic or optical permittivity, $\varepsilon_{\infty}$ (the square of the refractive index), if a nonequilibrium response for the environment is used. The presence of the solvent and the MM charges and dipoles of the bridge also affects the coupling in an implicit way, by affecting the D and A transition energies and densities and all the related transition properties. This is automatically accounted for by solving the LR equations, which now include explicit solvent- (PCM) and bridge- (MMPol) induced terms.

All the QM calculations were run at the TD-DFT level, employing the CAM-B3LYP functional and the 6-31G(d) basis set, using a locally modified version of the Gaussian09 suite of codes. ${ }^{25}$

The $\mathrm{MMPol}^{21}$ part of the system was described using fixed MM charges obtained from a fit of the electrostatic potential of the molecule or fragment according to the Merz and Kollman method $^{26,27}$ at the CAM-B3LYP/6-31G(d) level, plus a set of polarizable sites (coincident with the $\mathrm{MM}$ atoms) described by isotropic polarizabilities. We employed the Thole model, ${ }^{28}$ which avoids intramolecular overpolarization problems by using a smeared dipole-dipole interaction tensor. Atomic isotropic polarizability values were taken from the fit of experimental molecular polarizabilities performed by van Duijnen and Swart. ${ }^{29}$

The presence of covalent bonds between the QM and the MM fragments was tackled by following the link atom method: ${ }^{30}$ the QM-MM bonds were initially cut and saturated on both sides with hydrogens. The saturated MM fragment, isolated, was then used to obtain the Merz-Kollman charges; afterwards, the MM atoms previously bound to the QM chromophores, together with their saturation hydrogens, were removed to avoid hyperpolarization problems; their MM charges were summed and distributed onto the covalently bound MM atoms. 


\section{Results}

In all the systems here studied, energy transfer occurs from the photoexcited zinc-porphyrin (Zn, the donor) to the free base-porphyrin ( $\mathrm{Fb}$, the acceptor). The excitations involved are the (weak) Q bands, localized on the two porphyrins, which are virtually degenerate for the $\mathrm{Zn}$ porphyrin and slightly split for the $\mathrm{Fb}$ one. The comparison between the experimental absorption spectra of the isolated porphyrins and that of the $\mathrm{ZnFb}$ complex shows no marked modification, either in shape or in position, thus indicating a relatively weak electronic interaction. ${ }^{9}$ The spectra of the fluorinecontaining $\mathrm{Zn}$ porphyrins resemble those of their non-fluorinated counterparts, while the oscillator strength of the $\mathrm{S}_{0} \rightarrow$ Q-state transition in fluorinated $\mathrm{Fb}$ is reduced by $40 \%$ with respect to the non-fluorinated one.

\subsection{Excitation energies}

As a preliminary analysis we have compared experimental absorption maxima for the different porphyrins with the calculated vertical energies. All calculations have been performed on ground-state optimized structures and they include the effects of the toluene solvent through a PCM description. For this analysis three different basis sets have been compared, namely 6-31G(d), 6-31+G(d) and 6-311+G(d); the CAM-B3LYP functional has been employed.

Table 1: Experimental and calculated absorption wavelengths (nm) of the Q-states of $\mathrm{Zn}$ and $\mathrm{Zn}$ free-porphyrins in toluene. The calculated transition dipoles are also reported in brackets (a.u.). The experimental values are taken from Strachan et al. ${ }^{9}$ and Seth et al.. ${ }^{31}$

\begin{tabular}{lccccccc}
\hline Monomer & Expt. & \multicolumn{2}{c}{ Calc. 6-31G(d) } & \multicolumn{2}{c}{ Calc. 6-31+G(d) } & \multicolumn{2}{c}{ Calc. 6-311+G(d) } \\
\hline \multirow{2}{*}{$\mathrm{ZnU}$} & \multirow{2}{*}{551} & 528 & $(0.005)$ & 535 & $(0.010)$ & 538 & $(0.003)$ \\
& & 528 & $(0.006)$ & 536 & $(0.010)$ & 539 & $(0.017)$ \\
$\mathrm{FbU}$ & \multirow{2}{*}{516} & 527 & $(0.153)$ & 531 & $(0.136)$ & 535 & $(0.107)$ \\
& & 579 & $(0.073)$ & 581 & $(0.071)$ & 583 & $(0.053)$ \\
$\mathrm{F}_{15} \mathrm{ZnU}$ & \multirow{2}{*}{548} & 529 & $(0.000)$ & 537 & $(0.003)$ & 540 & $(0.012)$ \\
& & 530 & $(0.302)$ & 538 & $(0.408)$ & 542 & $(0.473)$ \\
$\mathrm{F}_{15} \mathrm{FbU}$ & \multirow{2}{*}{510} & 526 & $(0.186)$ & 531 & $(0.176)$ & 535 & $(0.171)$ \\
& & 575 & $(0.114)$ & 578 & $(0.157)$ & 581 & $(0.171)$ \\
$\mathrm{ZnB}$ & \multirow{2}{*}{551} & 529 & $(0.000)$ & 537 & $(0.000)$ & 541 & $(0.002)$ \\
& & 530 & $(0.043)$ & 538 & $(0.048)$ & 541 & $(0.028)$ \\
$\mathrm{FbB}$ & \multirow{2}{*}{516} & 528 & $(0.302)$ & 534 & $(0.276)$ & 538 & $(0.227)$ \\
& & 578 & $(0.140)$ & 581 & $(0.143)$ & 584 & $(0.114)$ \\
\hline
\end{tabular}


As shown in the Table, the QM/PCM approach well describes the relative positions of the Qbands for all porphyrins. As expected, the most extended basis set shows a better agreement with the experiments, but the variations are always quite limited both in wavelengths and in transition dipoles. For what concerns the latter ones, the enlargement of the basis set induces a small reduction of the transition dipoles for the $\mathrm{Zn}$ free-porphyrins which is however counterbalanced by a small increase for the Zn-porphyrins. On the basis of these results we have selected the 6-31G(d) basis set for the following analysis.

\subsection{Energy transfer}

According to the experimental analysis carried out by Strachan et al., ${ }^{9}$ the EET mechanism remains predominantly through-bond (TB) rather than through-space (TS) in all the systems, even if the rate is considerably slower in $\mathrm{F}_{30} \mathrm{ZnFbU}$ than in all the other arylethyne-linked $\mathrm{ZnFb}$ dimers. The observed energy transfer rate was therefore assumed to be due to the additive effects of the TB $\left(k_{\mathrm{TB}}\right)$ and TS $\left(k_{\mathrm{TS}}\right)$ processes, where the (approximate) Förster formula was used to calculate the TS transfer rate:

$$
k_{\mathrm{TS}}=k_{\mathrm{EET}}^{\text {Förster }}=8.785 \times 10^{-25} \frac{\kappa^{2} \Phi_{\mathrm{D}} I}{\tau_{\mathrm{D}} n^{4} R_{\mathrm{DA}}^{6}} .
$$

Here, $\kappa$ is an orientation factor, $\Phi_{\mathrm{D}}$ and $\tau_{\mathrm{D}}$ the fluorescence quantum yield and lifetime of the donor chromophore in the absence of the acceptor, $I$ the Förster spectral overlap term (in mmol ${ }^{-1}$ $\mathrm{cm}^{6}$ ), $n$ the solvent refractive index, and $R_{\mathrm{DA}}$ the donor-acceptor centre-to-centre distance (in $\mathrm{cm}$ ). The TB contribution was derived from the relations:

$$
\begin{aligned}
& \chi_{\mathrm{TS}}=\frac{k_{\mathrm{TS}}}{k_{\mathrm{tot}, \text { expt. }}} \\
& \chi_{\mathrm{TB}}+\chi_{\mathrm{TS}}=1
\end{aligned}
$$

and the results obtained in toluene are reported in Table 2.

As it can be seen from the Table, the TS energy transfer rates for all three dimers are relatively 
Table 2: Experimental results and Förster-extrapolated TS and TB contributions, from Strachan et al.. ${ }^{9}$ The spectral overlap $I$ is reported in $10^{14} \mathrm{mmol}^{-1} \mathrm{~cm}^{6}$ and the inverse rate constants $k^{-1}$ in ps.

\begin{tabular}{l|rrrrr}
\hline Dimer & $I$ & $k^{-1}$ & $k_{\mathrm{TS}}^{-1}$ & $\chi_{\mathrm{TB}}$ & $\chi_{\mathrm{TS}}$ \\
\hline $\mathrm{ZnFbU}$ & 2.94 & 24 & 745 & 0.96 & 0.04 \\
$\mathrm{~F}_{30} \mathrm{ZnFbU}$ & 1.88 & 240 & 1326 & 0.82 & 0.18 \\
$\mathrm{ZnFbB}\left(\mathrm{CH}_{3}\right)_{4}$ & 3.53 & 115 & 644 & 0.82 & 0.18 \\
\hline
\end{tabular}

slow and differ by less than $\sim 2$-fold. $\mathrm{F}_{30} \mathrm{ZnFbU}$ exhibits the slowest $\mathrm{TS}$ rate owing to the low fluorescence yield of the $\mathrm{Zn}$ porphyrin and the diminished oscillator strength of the ground $\rightarrow$ Qexcited-state absorption transition of the $\mathrm{Fb}$ porphyrin. The resulting $\mathrm{TB}$ energy transfer rate for $\mathrm{F}_{30} \mathrm{ZnFbU}$ accounts for $\sim 82 \%$ of the total energy transfer. Accordingly, the TB energy transfer rate is $\sim 12$-fold slower in $\mathrm{F}_{30} \mathrm{ZnFbU}$ than in $\mathrm{ZnFbU}$. Note that the reduced spectral overlap, which also contributes to the TB mechanism, can only account for $15-20 \%$ of the attenuation in the energy transfer rate observed when passing from $\mathrm{ZnFbU}$ to $\mathrm{F}_{30} \mathrm{ZnFbU}$. This is due to the fact that the spectral overlap integrals for this process, like those for the TS mechanism, differ by less than 2-fold.

In order to assess the influence of the bridge on the coupling, we have built three models following what we did in a previous paper: ${ }^{19}$ (a) model M0, a full-QM approach which completely neglects the bridge; (b) model Mc, again a full-QM approach, which includes the entire bridge at QM level; (c) model MMPol, a hybrid QM/MM where the the QM part coincides with the M0 but the bridge is here described using the polarizable MM approach presented above (see Figure 2). We note that in the Mc model the partition into donor and acceptor can be done in various ways; consistently with the previous paper, we associate the phenyl-ethyne segment of the bridge to the donor Zn-porphyrin, and the other phenyl segment to the $\mathrm{Fb}$-porphyrin, although the results obtained do not change if the bridge is partitioned otherwise.

For the $\mathrm{F}_{30} \mathrm{ZnFbU}$ and $\mathrm{ZnFbU}$ dimers, two different conformers are possible, even if the orientation of the lateral groups is kept fixed: the two porphyrins can either lie on the same plane or be tilted (the optimized tilt angle is $41^{\circ}$ for $\mathrm{F}_{30} \mathrm{ZnFbU}$ and $43^{\circ}$ for $\mathrm{ZnFbU}$ ). The coplanar conformers will be henceforth labelled 'P', and the tilted ones ' $\mathrm{T}$ '. For what concerns the $\mathrm{ZnFbB}\left(\mathrm{CH}_{3}\right)_{4}$ 


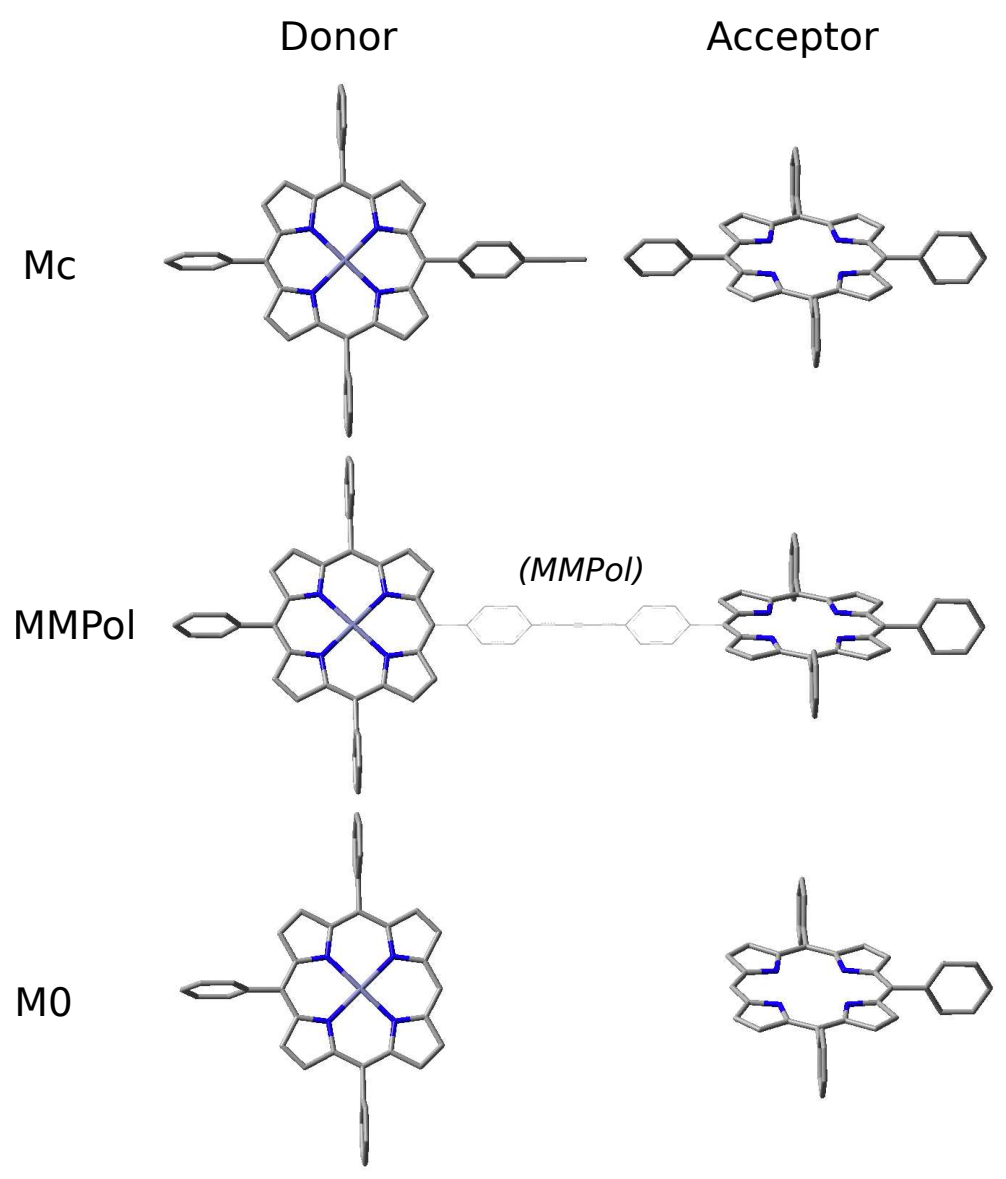

Figure 2: The three models employed. The donor and acceptor moieties also include parts of the bridge in the full-QM model Mc, partitioned as shown. The bridge is not included in the full-QM model M0, while it is included as a set of charges and polarizable sites according to the MMPol method in the MMPol model. The $\mathrm{Zn}$ - and $\mathrm{Fb}$-porphyrins are always described quantummechanically.

dimer, the only characterized by a branched linker, there is only one possible conformer, with the porphyrin rings almost coplanar, and at the same time perpendicular to the plane containing the linker.

For the resulting five conformers, we have calculated the electronic coupling using the three models M0, Mc and MMPol, both in vacuo and in toluene. These are reported in Table 3 in terms of the sum of the squared couplings for each pair of excitations involving the two quasi-degenerate $\mathrm{Q}$ states (first and second excited states for both $\mathrm{D}$ and A moieties), i.e., $V^{2}=V_{1 \rightarrow 1}^{2}+V_{1 \rightarrow 2}^{2}+V_{2 \rightarrow 1}^{2}+V_{2 \rightarrow 2}^{2}$ (the indices refer to the excitation states of the donor and the acceptor, respectively). For the systems with two conformers ('P' and ' $\mathrm{T}$ '), a Boltzmann average has been computed in order to 
obtain effective couplings. For this purpose, the free energy of the monomers has been calculated in vacuo, so the weights in vacuo and in toluene are assumed to be the same (this is indeed a reasonable approximation due to the very low polarity of toluene).

Table 3: Squared couplings, calculated for the dimeric systems studied, both in vacuo and in toluene, using the MO, Mc and MMPol models. Data are reported in units of $\left(\mathrm{cm}^{-1}\right)^{2}$.

\begin{tabular}{lcc|rrr|rrr}
\hline \multirow{2}{*}{ Dimer } & & & \multicolumn{3}{|c|}{$V^{2}$, Vacuum } & \multicolumn{3}{c}{$V^{2}$, Toluene } \\
& Config. & Weight & M0 & MMPol & Mc & M0 & MMPol & Mc \\
\multirow{2}{*}{$\mathrm{ZnFbU}$} & $\mathrm{P}$ & 0.57 & $<0.1$ & 8.6 & 88.2 & $<0.1$ & 2.3 & 43.2 \\
& $\mathrm{~T}$ & 0.43 & $<0.1$ & 8.4 & 89.5 & $<0.1$ & 2.2 & 43.9 \\
& Weighted average & $<0.1$ & 8.5 & 88.7 & $<0.1$ & 2.3 & 43.5 \\
\hline \multirow{2}{*}{$\mathrm{F}_{30} \mathrm{ZnFbU}$} & $\mathrm{P}$ & 0.18 & 0.5 & 0.3 & 7.5 & 0.8 & 0.5 & 3.7 \\
& $\mathrm{~T}$ & 0.82 & 0.4 & 0.3 & 5.3 & 0.7 & 0.4 & 2.6 \\
& Weighted average & 0.4 & 0.3 & 5.7 & 0.7 & 0.4 & 2.8 \\
\hline \multirow{2}{*}{$\mathrm{H}_{30} \mathrm{ZnFbU}$} & $\mathrm{P}$ & & $<0.1$ & 8.8 & 84.6 & $<0.1$ & 3.0 & 48.2 \\
& $\mathrm{~T}$ & & $<0.1$ & 8.2 & 69.3 & $<0.1$ & 2.7 & 39.2 \\
\hline $\mathrm{ZnFbB}\left(\mathrm{CH}_{3}\right)_{4}$ & \multicolumn{2}{c|}{} & $<0.1$ & 9.2 & 20.2 & $<0.1$ & 4.5 & 12.6 \\
\hline
\end{tabular}

We first analyse the results relative to the Mc model, for all dimers, both in vacuo and in toluene. Concerning the effect of the conformation, we note that it only slightly affects the couplings. Moreover, we note that the calculated couplings for the $\mathrm{ZnFbU}$ dimer are quite larger than those for the other two systems. In particular, the squared couplings calculated for the fluorinated dimer $\mathrm{F}_{30} \mathrm{ZnFbU}$ are approximately one order of magnitude smaller than those for the non-fluorinated $\mathrm{ZnFbU}$, even if they have exactly the same linker. This behaviour, observed both in vacuo and in toluene, is in agreement with the experimental observations reported in Table 2, where the $\mathrm{ZnFbU}$ system shows the fastest experimental EET rate $\left(41.7 \mathrm{~ns}^{-1}\right)$, compared to those of $\mathrm{ZnFbB}\left(\mathrm{CH}_{3}\right)_{4}$ $\left(8.7 \mathrm{~ns}^{-1}\right)$ and $\mathrm{F}_{30} \mathrm{ZnFbU}\left(4.2 \mathrm{~ns}^{-1}\right)$, the latter being one order of magnitude slower than its nonfluorinated counterpart. Below, we will try to understand why and how the presence of the F atoms affects so markedly the electronic coupling and therefore the EET rate.

For what concerns the effect of the solvent, this is homogeneous in the three systems and it always leads to a significant reduction of the coupling, due to a dominant screening effect: the reduction is $\sim 30 \%$ for both fluorinated and non fluorinated $\mathrm{ZnFBU}$ and $\sim 20 \%$ for $\mathrm{ZnFbB}\left(\mathrm{CH}_{3}\right)_{4}$. The smaller screening in the $\mathrm{ZnFbB}\left(\mathrm{CH}_{3}\right)_{4}$ system is probably due to the fact that the lateral groups in the linker make the PCM cavity larger than in the other two systems; as a result, the solvent effect 
is reduced and so is the screening.

We now focus on the results obtained from the three different models Mc, M0 and MMPol. From the comparison between the two full-QM models, M0 and Mc, we note that the changes induced by the inclusion of the bridge in the Mc description are very marked, leading to a huge increase of the electronic coupling, particularly for the $\mathrm{ZnFbU}$ dimer. This shows that the coupling among the Q-excitations in all the systems studied is largely dominated by the presence of the linker. Note however that the effects of the same linker are different for different porphyrins: while $V^{2}$ in vacuo is almost null for the M0 model of both the fluorinated and non-fluorinated $\mathrm{ZnFbU}$ systems (having the same linker), these square couplings become $\sim 90$ and $\sim 6\left(\mathrm{~cm}^{-1}\right)^{2}$ when the Mc model is employed. This shows that it is the combination of the type of linker and the type of porphyrin which finally determines the strength of the interaction. The same behaviour can be observed in toluene, where however, as discussed above, the coupling values are always smaller.

Finally, the comparison between Mc and MMPol, and that between M0 and MMPol models, allow us to better understand the role of the linker, and the nature of its effect on the coupling. As a matter of fact, the MMPol model properly takes into account all the polarization effects of the linker, but cannot include eventual quantum-mechanical effects, such as those leading to the superexchange mechanism, which are, conversely, correctly accounted for in our Mc model.

From the data reported in Table 3, comparing M0 and MMPol results, we note that the fluorinated system does not show any real enhancement of the coupling due to polarization effects from the linker: quite the opposite, as the coupling seems to be screened by its polarization, both in vacuo and in toluene. On the other hand, we report a non negligible increase of the coupling when the MMPol model is employed for non fluorinated systems $\mathrm{ZnFbU}$ and $\mathrm{ZnFbB}\left(\mathrm{CH}_{3}\right)_{4}$, with the latter showing the largest polarization effect. Once again the same picture is maintained in toluene.

A better appreciation of the differences in the coupling and in the relative effects of the linker can be obtained by looking at the transition densities: the plots for each excitation of the donor (Zn-) porphyrin are reported in Figure 3.

It is interesting to observe that the largest contribution from the linker is found in the fluorinated 


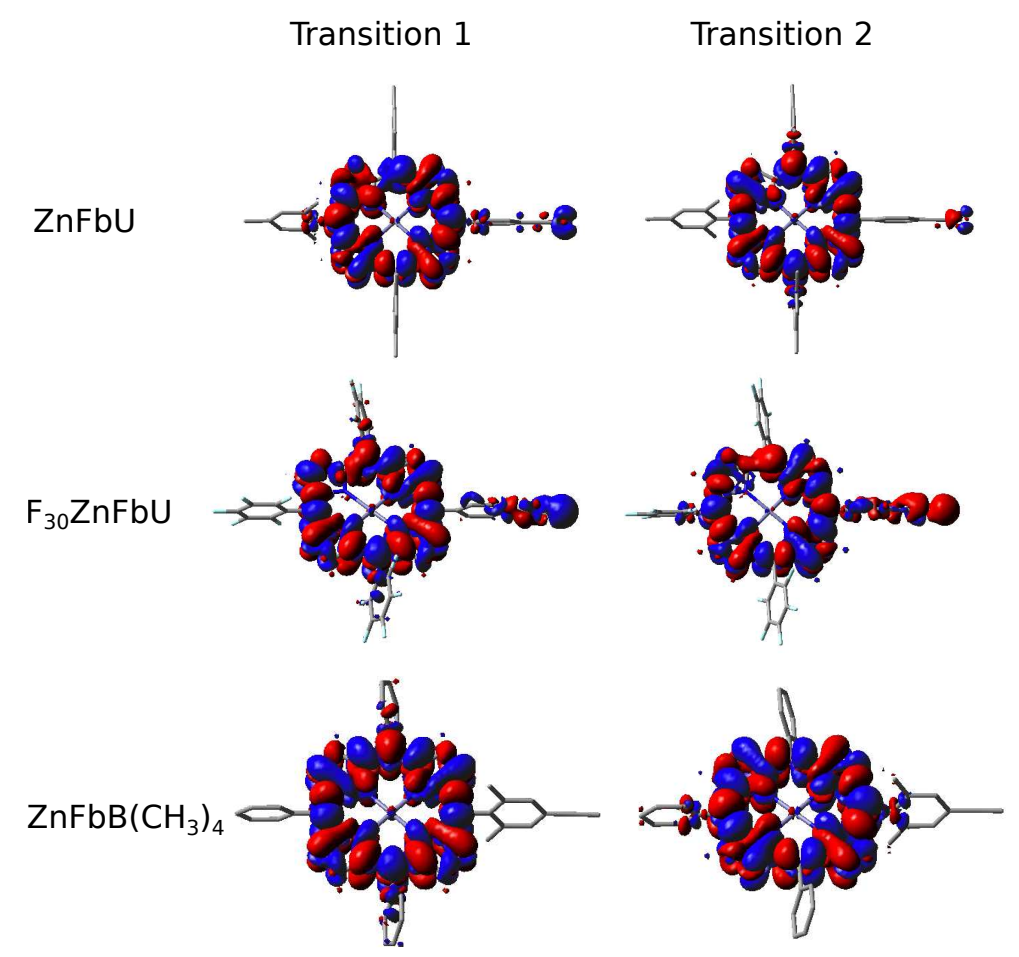

Figure 3: Transition densities relative to the first two excited states of the $\mathrm{Zn}$-porphyrin, calculated for the three dimers according to the Mc model.

system: e.g. the system for which the polarization effects of the linker are the lowest.

To analyse this and the other specificities of the fluorinated system with respect to the nonfluorinated ones and especially to the parallel $\mathrm{ZnFbU}$ system which shares the same linker, in the following section we examine both the structural and the electronic modifications induced by fluorination.

\subsection{Effect of fluorination}

In order to clarify whether the differences between the $\mathrm{ZnFbU}$ and $\mathrm{F}_{30} \mathrm{ZnFbU}$ dimers can be explained in terms of different optimized geometries, or if they are due to a distortion of the chromophores electron densities induced by the F atoms, we have inspected the transition dipole moments of three systems: the $\mathrm{ZnFbU}$ and $\mathrm{F}_{30} \mathrm{ZnFbU}$ dimers already studied, and a new dimer obtained from the $\mathrm{F}_{30} \mathrm{ZnFbU}$ equilibrium geometry, where the $\mathrm{F}$ atoms have been replaced by $\mathrm{H}$ atoms, appropriately reducing the $\mathrm{C}-\mathrm{F}$ bond length. The squared couplings for this dimer, la- 
belled $\mathrm{H}_{30} \mathrm{ZnFbU}$ in Table 3, are remarkably similar to those of $\mathrm{ZnFbU}$. We therefore conclude that the effect of the $\mathrm{F}$ atoms does not lie in the distortion of the nuclear geometry, but rather in the perturbation of the electron density.

In Table 4 we report the transition dipoles of the donor and acceptor chromophores, and the orientation factor $\kappa$, for some systems, calculated in vacuo. The factor $\kappa$ is helpful to understand how the mutual orientation of these dipoles affects the coupling, in a Förster-like, dipole-dipole interaction picture. The Förster coupling in vacuo is calculated as:

$$
V^{(\mathrm{F})} \approx\left[\hat{\mu}_{\mathrm{D}}^{\mathrm{T}} \cdot \hat{\mu}_{\mathrm{A}}^{\mathrm{T}}-3\left(\hat{\mu}_{\mathrm{D}}^{\mathrm{T}} \cdot \hat{R}_{\mathrm{DA}}\right)\left(\hat{\mu}_{\mathrm{A}}^{\mathrm{T}} \cdot \hat{R}_{\mathrm{DA}}\right)\right] \frac{\mu_{\mathrm{D}}^{\mathrm{T}} \mu_{\mathrm{A}}^{\mathrm{T}}}{R_{\mathrm{DA}}^{3}}
$$

where $\vec{\mu}_{\mathrm{D}}^{\mathrm{T}}$ and $\vec{\mu}_{\mathrm{A}}^{\mathrm{T}}$ are the donor and acceptor transition dipoles, respectively, and $\vec{R}_{\mathrm{DA}}$ the donoracceptor vector distance; the terms in square brackets correspond to the definition of the orientation factor $\kappa$.

Table 4: Donor and acceptor transition dipole moments (in a.u.), orientation factors and squared couplings (in $\mathrm{cm}^{-2}$ ) calculated in vacuo and in toluene, using the MO, Mc and MMPol models. The indices of $\kappa$ refer to the corresponding donor and acceptor states.

\begin{tabular}{l|rrrr|rrrr|c}
\hline Dimer & $\mu_{\mathrm{D}, 1}^{\mathrm{T}}$ & $\mu_{\mathrm{D}, 2}^{\mathrm{T}}$ & $\mu_{\mathrm{A}, 1}^{\mathrm{T}}$ & $\mu_{\mathrm{A}, 2}^{\mathrm{T}}$ & $\left|\kappa_{1,1}\right|$ & $\left|\kappa_{1,2}\right|$ & $\left|\kappa_{2,1}\right|$ & $\left|\kappa_{2,2}\right|$ & $V^{2(\mathrm{~F})}$ \\
\hline $\mathrm{ZnFbU} \mathrm{(P)}$ & 0.289 & 0.129 & 0.355 & 0.547 & 1.53 & 1.54 & 0.65 & 0.63 & 1.62 \\
$\mathrm{H}_{30} \mathrm{ZnFbU}(\mathrm{P})$ & 0.322 & 0.206 & 0.354 & 0.597 & 1.50 & 1.42 & 0.56 & 0.80 & 2.17 \\
$\mathrm{~F}_{30} \mathrm{ZnFbU}(\mathrm{P})$ & 0.102 & 0.116 & 0.179 & 0.401 & 0.88 & 0.83 & 0.79 & 1.00 & 0.07 \\
\hline
\end{tabular}

The data reported in Table 4, although quantitatively approximate since relying on a dipoledipole approximation, is however useful to show that the effect of the fluorination is dual; on one hand it causes a reduction of the transition dipole moments: see for instance $\mu_{\mathrm{D}, 1}^{\mathrm{T}}$ and $\mu_{\mathrm{A}, 1}^{\mathrm{T}}$, that are reduced by nearly 3- and 2-fold, respectively, when passing from non-fluorinated systems to $\mathrm{F}_{30} \mathrm{ZnFbU}$. At the same time, the absolute value of the orientation factors involving the first state of the donor $\left(\kappa_{1,1}\right.$ and $\left.\kappa_{1,2}\right)$ are also markedly reduced.

Even in such a simplistic picture, the combination of the two effects explains fairly well the observed reduction of the squared couplings. The remarkably consistent results of the two nonfluorinated systems $\mathrm{ZnFbU}$ and $\mathrm{H}_{30} \mathrm{ZnFbU}$, despite their different structure, point out that such a 
reduction can be explained in terms of the perturbation of the chromophore electron densities due to the presence of the $\mathrm{F}$ atoms.

\subsection{Through-space and through-bond contributions}

Finally, we move to the comparison with the experimental data reported in Table 2. To do that we define the TS contribution to the total coupling, relative to a method M, as the fraction of the squared coupling obtained with that method, relative to the squared coupling obtained with the most complete Mc method, namely:

$$
\chi_{\mathrm{TS}}^{(\mathrm{M})} \equiv \frac{\left|V^{(\mathrm{M})}\right|^{2}}{\left|V^{(\mathrm{Mc})}\right|^{2}} .
$$

We have computed the TS contribution for the M0 and MMPol models, both in vacuo and in toluene. The comparison with the results by Strachan et al. ${ }^{9}$ for the three porphyrin dimers under study are reported in Table 5. For $\mathrm{ZnFbU}$ and $\mathrm{F}_{30} \mathrm{ZnFbU}$, the Boltzmann average of the results relative to the two conformers is shown.

Table 5: Percent of the TS contribution to the total coupling, $\chi_{\mathrm{TS}}$. (a) The experimental values, from Strachan et al., ${ }^{9}$ are calculated in a Förster-type approximation.

\begin{tabular}{lrrrr}
\hline & $\chi_{\mathrm{TS}}^{(\mathrm{M} 0, \mathrm{vac})}$ & $\chi_{\mathrm{TS}}^{(\mathrm{MMPol}, \mathrm{vac})}$ & $\chi_{\mathrm{TS}}^{(\text {MMPol,tol })}$ & \multicolumn{1}{c}{$\chi_{\mathrm{TS}}^{\text {Expt. }}(\mathrm{a})$} \\
\hline $\mathrm{ZnFbU}$ & $<1 \%$ & $10 \%$ & $5 \%$ & $4 \%$ \\
$\mathrm{~F}_{30} \mathrm{ZnFbU}$ & $7 \%$ & $5 \%$ & $16 \%$ & $18 \%$ \\
$\mathrm{ZnFbB}\left(\mathrm{CH}_{3}\right)_{4}$ & $<1 \%$ & $46 \%$ & $36 \%$ & $18 \%$ \\
\hline
\end{tabular}

The data in Table 5 shows that the estimates from the M0 model are completely off the Förster analysis, with their TS contributions being almost null for $\mathrm{ZnFbU}$ and $\mathrm{ZnFbB}\left(\mathrm{CH}_{3}\right)_{4}$, and very low for $\mathrm{F}_{30} \mathrm{ZnFbU}$. For what concerns the MMPol estimates, on the other hand, the results show a non negligible contribution of the TS interaction, particularly for the $\mathrm{ZnFbB}\left(\mathrm{CH}_{3}\right)_{4}$ system. By introducing the solvent effects, $\chi_{\mathrm{TS}}$ significantly changes for all the systems. These changes are different both in module and in sign for the three systems: we observe a net increase of the TS contribution for $\mathrm{F}_{30} \mathrm{ZnFbU}$, from 5 to $16 \%$, bringing our results closer to the $18 \%$ contribution derived 
experimentally. On the other hand, the TS contribution decreases markedly for the ZnFbU system, and even more so for the $\mathrm{ZnFbB}\left(\mathrm{CH}_{3}\right)_{4}$ dimer. For these also we note that the solvent-induced changes lead towards a much better agreement with the Förster estimates from experimental data, but while the agreement in absolute terms is fairly good for the $\mathrm{ZnFbU}$ and $\mathrm{F}_{30} \mathrm{ZnFbU}$ dimers, our calculated TS contribution is twice the Förster one in the case of the $\mathrm{ZnFbB}\left(\mathrm{CH}_{3}\right)_{4}$ dimer.

In order to better understand the origin of such differences in the results for the different systems, we have further investigated the possible role played by torsional motions of the planes of the two porphyrins with respect to that of the linker.

\subsection{Twisting motion}

Several geometries of the $\mathrm{ZnFbB}\left(\mathrm{CH}_{3}\right)_{4}$ and $\mathrm{ZnFbU}$ dimers (the latter in its planar configuration) have been analysed. The torsion angles between the Donor-Bridge and Acceptor-Bridge planes were artificially twisted from their equilibrium value. Note that the phenylene flexibility in oligo(phenyleneethynylene) (OPE) linkers like ours has been found to affect the energy and electron transfer in several studies (see for instance Albinsson and Mårtensson ${ }^{32}$ ). In particular, we have considered small variations of $15^{\circ}$ maximum, obtained by rotating the porphyrin planes in various ways. We have identified each configuration by the dihedral angles $\phi_{\mathrm{Zn}}, \phi_{\mathrm{Fb}}$ and $\psi$, defined as those between $\mathrm{D}$ and $\mathrm{B}, \mathrm{A}$ and $\mathrm{B}$, and $\mathrm{D}$ and $\mathrm{A}$, respectively; we have also defined $\Delta \phi_{\mathrm{Zn}}, \Delta \phi_{\mathrm{Fb}}$ and $\Delta \psi$ as their variation with respect to the equilibrium value.

The four possible combined twisting motions have been named 'Zn', 'Fb', 'Syn' and 'Anti' (see Figure 5). For each one there are two possible resulting configurations, depending on the positive or negative direction of the rotation, and they are labelled $(+)$ and $(-)$. The results obtained, in vacuo and in toluene, for the squared couplings (Mc and MMPol models), and the relative TS contributions, are reported in Tables $6\left(\mathrm{ZnFbB}\left(\mathrm{CH}_{3}\right)_{4}\right)$ and $7(\mathrm{ZnFbU})$.

We first analyse the results for the $\mathrm{ZnFbB}\left(\mathrm{CH}_{3}\right)_{4}$ dimer, shown in Table 6. Note that, because of the symmetrical structure characterized by almost coplanar porphyrins, that are conversely almost perpendicular to the bridge plane $\left(\phi_{\mathrm{Zn}}=90.7^{\circ}, \phi_{\mathrm{Fb}}=94.1^{\circ}, \psi=-4.6^{\circ}\right)$, the results are indepen- 


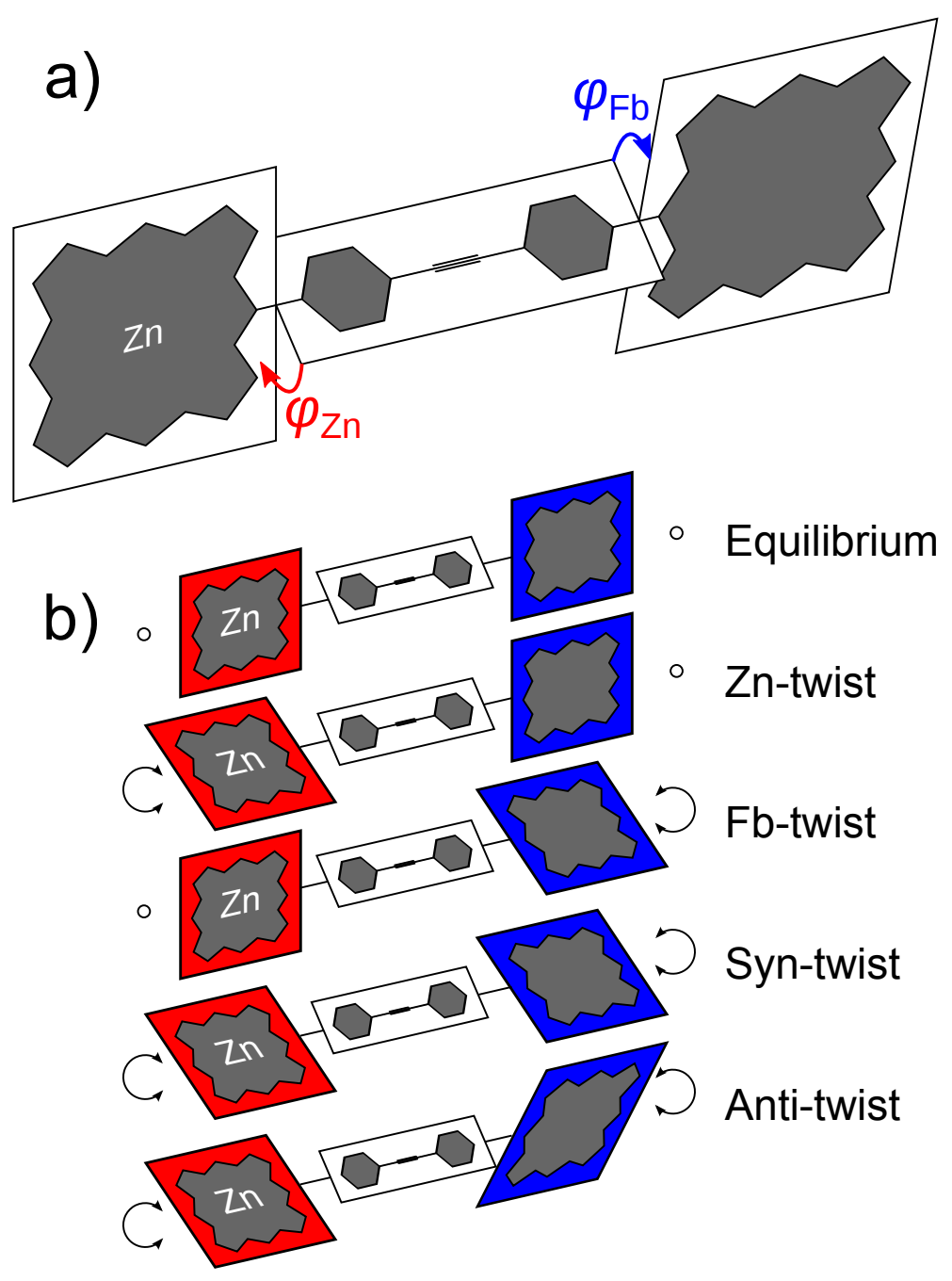

Figure 4: Schematic representation of: (a) the donor, bridge and acceptor planes, and the $\phi_{\mathrm{Zn}}$ and $\phi_{\mathrm{Fb}}$ angles; (b) the four possible twisting motions here considered.

dent of the twisting direction and in the Table we only report a single set of twisting angles.

For what concerns the couplings obtained with the complete $\mathrm{Mc}$ model, $V^{2(\mathrm{Mc})}$, we observe that the rotation of the $\mathrm{Zn}$-porphyrin seems to lead to somewhat larger changes than the corresponding rotation in the Fb-porphyrin: compare for instance the $\mathrm{Zn}$ - and Fb-twists, both characterized by a variation of $15^{\circ}$, where the couplings increase by $65 \%$ in the first case, and only by $34 \%$ in the second. The rotation of both porphyrins finally leads to a more than doubling of the squared coupling. The same trend can be seen in toluene. In general, we see that small variations in the dihedral angles can lead to variations of the total coupling and, consequently, of the resulting EET rate. 
Table 6: Squared couplings, in vacuo and toluene, calculated using the Mc and MMPol models, and relative TS contribution, for the configurations obtained by twisting the dihedral angles of $\mathrm{ZnFbB}\left(\mathrm{CH}_{3}\right)_{4}$ from its equilibrium position. The equilibrium values are: $\phi_{\mathrm{Zn}}=$ $90.7^{\circ}, \phi_{\mathrm{Fb}}=94.1^{\circ}, \psi=-4.6^{\circ}$.

\begin{tabular}{lrrrr|ccc|ccc}
\hline & & \multicolumn{3}{c|}{ Torsion angle variation } & \multicolumn{4}{c|}{ Vacuum } & \multicolumn{3}{c}{ Toluene } \\
\multicolumn{2}{l}{ Twisting } & $\Delta \phi_{\mathrm{Zn}}$ & $\Delta \phi_{\mathrm{Fb}}$ & $\Delta \psi$ & $V^{2(\mathrm{Mc})}$ & $V^{2}(\mathrm{MMPol})$ & $\chi_{\mathrm{TS}}^{(\mathrm{MMPol})}$ & $V^{2(\mathrm{Mc})}$ & $V^{2(\mathrm{MMPol})}$ & $\chi_{\mathrm{TS}}^{(\mathrm{MMPol})}$ \\
\hline Equilibrium & 0.0 & 0.0 & 0.0 & 20.2 & 9.2 & $46 \%$ & 12.6 & 4.5 & $36 \%$ \\
$\mathrm{Zn}$ & $(+)$ & 15.0 & 0.0 & 15.0 & 33.4 & 8.0 & $24 \%$ & 21.3 & 4.0 & $19 \%$ \\
$\mathrm{Fb}$ & $(+)$ & 0.0 & 15.0 & 15.0 & 27.0 & 8.6 & $32 \%$ & 17.1 & 4.3 & $25 \%$ \\
Syn & $(+)$ & 15.0 & 15.0 & 0.0 & 46.0 & 7.5 & $16 \%$ & 29.9 & 3.8 & $13 \%$ \\
Anti & $(+)$ & 7.5 & -7.5 & -15.0 & 24.8 & 8.7 & $35 \%$ & 15.7 & 4.4 & $28 \%$ \\
\hline
\end{tabular}

The MMPol squared couplings for the same system, $V^{2}$ (MMPol), show a different, smaller dependence on the dihedral angle; in general, any twisting motion results in a slightly smaller MMPol coupling than in the equilibrium configuration. The TS percent, calculated according to Equation 7 , is greatly affected by the dependence of its denominator on the twisting angle, and is consequently reduced with respect to the equilibrium value. Interestingly, the TS percent in toluene, $\chi_{\mathrm{TS}}^{(\mathrm{MMPol}, \mathrm{tol})}$, reduces in two cases ( $\mathrm{Zn}$ - and Syn-twist motions) to 19 and $13 \%$, respectively, much closer to the experimental $18 \%$ than the equilibrium value. It is perhaps possible to explain the discrepancy observed in Table 5 between our values and the Förster-derived ones, for the $\mathrm{ZnFbB}\left(\mathrm{CH}_{3}\right)_{4}$ dimer (36 vs. 18\%), in terms of twisted configurations from the vibrational motion contributing towards the experimental value.

Table 7: Squared couplings, in vacuo and toluene, calculated using the Mc and MMPol models, and relative TS contribution, for the configurations obtained by twisting the dihedral angles of $\mathrm{ZnFbBU}$ from its equilibrium position. The equilibrium values are: $\phi_{\mathrm{Zn}}=68.3^{\circ}$, $\phi_{\mathrm{Fb}}=67.1^{\circ}, \psi=1.2^{\circ}$.

\begin{tabular}{lcrrr|rrr|rrr}
\hline & \multicolumn{6}{c|}{ Torsion angle variation } & \multicolumn{3}{c|}{ Vacuum } & \multicolumn{3}{c}{ Toluene } \\
\multicolumn{2}{c}{ Twisting } & $\Delta \phi_{\mathrm{Zn}}$ & $\Delta \phi_{\mathrm{Fb}}$ & $\Delta \psi$ & $V^{2(\mathrm{Mc})}$ & $V^{2}(\mathrm{MMPol})$ & $\chi_{\mathrm{TS}}^{(\mathrm{MMPol})}$ & $V^{2(\mathrm{Mc})}$ & $V^{2(\mathrm{MMPl})}$ & $\chi_{\mathrm{TS}}^{(\mathrm{MMPol})}$ \\
\hline \multicolumn{2}{c}{ Equilibrium } & 0.0 & 0.0 & 0.0 & 88.2 & 8.6 & $10 \%$ & 43.2 & 2.3 & $5 \%$ \\
$\mathrm{Zn}$ & $(+)$ & 15.0 & 0.0 & 15.0 & 53.9 & 9.5 & $18 \%$ & 25.0 & 2.6 & $10 \%$ \\
& $(-)$ & -15.0 & 0.0 & -15.0 & 164.8 & 7.0 & $4 \%$ & 85.1 & 1.9 & $2 \%$ \\
\multirow{2}{*}{$\mathrm{Fb}$} & $(+)$ & 0.0 & 15.0 & 15.0 & 39.8 & 9.9 & $25 \%$ & 17.9 & 2.7 & $15 \%$ \\
& $(-)$ & 0.0 & -15.0 & -15.0 & 218.1 & 6.4 & $3 \%$ & 115.3 & 1.7 & $1 \%$ \\
\multirow{2}{*}{ Syn } & $(+)$ & 15.0 & 15.0 & 0.0 & 24.5 & 11.1 & $45 \%$ & 10.5 & 3.1 & $29 \%$ \\
& $(-)$ & -15.0 & -15.0 & 0.0 & 409.2 & 5.3 & $1 \%$ & 228.3 & 1.4 & $1 \%$ \\
\multirow{2}{*}{ Anti } & $(+)$ & 7.5 & -7.5 & -15.0 & 76.9 & 8.6 & $11 \%$ & 35.4 & 2.3 & $7 \%$ \\
\multirow{2}{*}{ Anti } & $(-)$ & -7.5 & 7.5 & -15.0 & 105.7 & 8.0 & $8 \%$ & 52.3 & 2.1 & $4 \%$ \\
& $(+)$ & 15.0 & -15.0 & -30.0 & 74.0 & 8.1 & $11 \%$ & 35.0 & 2.2 & $6 \%$ \\
& $(-)$ & -15.0 & 15.0 & -30.0 & 132.8 & 7.1 & $5 \%$ & 66.4 & 1.9 & $3 \%$ \\
\hline
\end{tabular}


The second dimer, $\mathrm{ZnFbU}$, for which a good agreement was found between the experimental and calculated $\chi_{\mathrm{TS}}$ at the equilibrium configuration, is characterized by a less symmetric starting geometry $\left(\phi_{\mathrm{Zn}}=68.3^{\circ}, \phi_{\mathrm{Fb}}=67.1^{\circ}, \psi=1.2^{\circ}\right)$ : the porphyrins are almost coplanar, but their angle with the bridge plane is far from being perpendicular.

As shown in Table 7, a much more drastic change of the squared couplings calculated with the Mc model if found for this system: in vacuo, the $\operatorname{Syn}(-)$ twist causes a nearly 5-fold enhancement, while conversely the opposite $\operatorname{Syn}(+)$ twist a nearly 4-fold reduction, in vacuo. In general, any twisting motion generates an enhancement with respect to the equilibrium value when it is applied in one direction, and a reduction in the opposite direction.

The squared couplings calculated with the MMPol model, on the other hand, are not only relatively less affected by the changes in geometry, but their variation is opposite to that obtained with the Mc model. The resulting behaviour of the TS contribution $\chi_{\mathrm{TS}}^{(\mathrm{MMPol})}$, calculated in toluene, is shown in Figure 5.

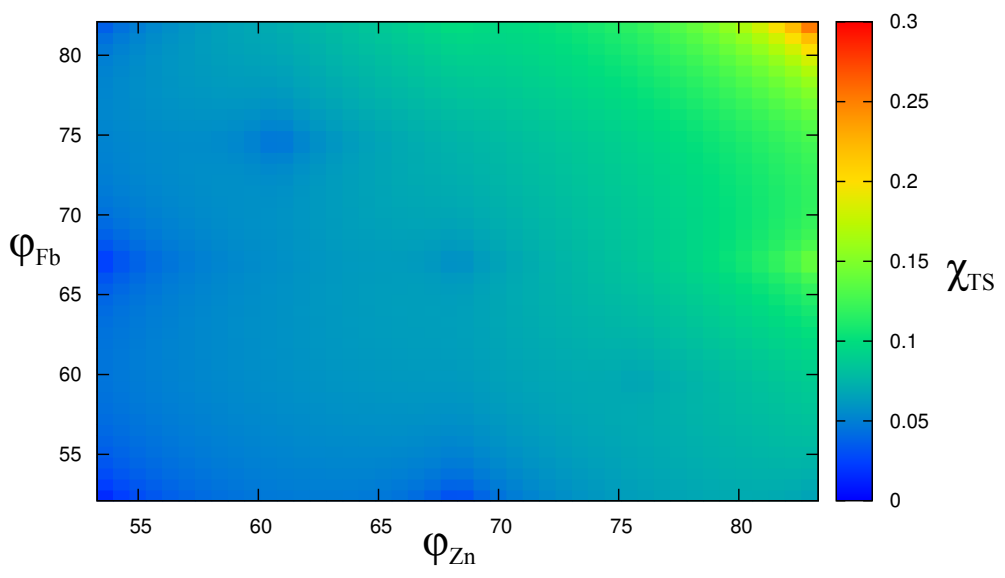

Figure 5: Colour map of the TS contribution for the $\mathrm{ZnFbU}$ dimer, $\chi_{\mathrm{TS}}^{(\mathrm{MMPol})}$, calculated in toluene using the MMPol model. The equilibrium angles are $68.3^{\circ}$ and $67.1^{\circ}$.

From such a graph, it is clear that the TS contribution is higher the closer $\left(\phi_{\mathrm{Zn}}, \phi_{\mathrm{Fb}}\right)$ is to $\left(90^{\circ}, 90^{\circ}\right)$. A similar behaviour can be observed for the $\mathrm{ZnFbB}\left(\mathrm{CH}_{3}\right)_{4}$ dimer (plot not shown), where the equilibrium value $\left(90.7^{\circ}, 94.1^{\circ}\right)$ is almost at the maximum.

From the analysis of the two systems, we can say that, statistically, the torsional motion around the equilibrium geometry value reduces the TS contribution for $\mathrm{ZnFbB}\left(\mathrm{CH}_{3}\right)_{4}$, while it leaves the 
one calculated for $\mathrm{ZnFbU}$ virtually unaltered. In both cases, the calculated results tend to agree with the experimental observation.

\section{Conclusion}

We have studied three $\mathrm{ZnFb}$ porphyrin dimers linked by a semirigid phenyleneethynylene spacer, focusing on the effects that different linkers and substituents have on the electronic coupling, and comparing our results, when possible, to the experimental values by Strachan et al.. ${ }^{9}$

We have employed our mixed QM/discrete/continuum method, where the QM parts of the system are described at the TD-DFT level, the discrete ones following the MMPol classical polarizable description, and the solvent is introduced as a structureless continuum. Employing models which differentiates by the way the linker is accounted for, has allowed us to estimate the through-bond and through-space contributions to the total coupling.

Our results are in general good agreement with the experimental ones, and in particular we verify the the fluorination strongly affects the electronic communication among the dimer moieties, despite the fact that the system geometry is mostly unaltered. We also obtain values for the TS contribution to the total coupling that are in agreement with the experimental ones, particularly for the two systems $\mathrm{ZnFbU}$ and $\mathrm{F}_{30} \mathrm{ZnFbU}$, characterized by the same linker. For the third system, $\mathrm{ZnFbB}\left(\mathrm{CH}_{3}\right)_{4}$, our results on the TB contribution seem to indicate that the twisting motion of the porphyrin planes with respect to the bridge plane may play a more relevant role than one would expect, particularly if we consider that the methyl groups on the linker make this structure more rigid than the others.

In general, our approach performs fairly well in describing the porphyrin dimers examined. As predicted in several experimental and theoretical studies on similar systems, the highly conjugated linker greatly affects the electronic communication between the energy donor and acceptor, mostly through a superexchange mechanism involving the bridge orbitals. This, on one hand, poses a serious limit to a QM/discrete description of the system, since the bridge polarization has only a 
marginal effect on the total coupling. Our MMPol model, therefore, cannot be expected to provide a correct description of the energy transfer process, as it did with other systems studied elsewhere (see for instance the PDI-TDI dimer in Curutchet et al. ${ }^{15}$ and Caprasecca et al. ${ }^{19}$ ). On the other hand, when the results from the MMPol model are compared to those given by the full-QM model, Mc, we are able to estimate the TB and TS contributions and evaluate how these are affected by, for instance, structural modifications or solvation. Finally, it is important to stress that this last term, solvation, introduced through the PCM method, again proves essential to correctly describe real systems and obtain reasonable results.

\section{References}

(1) Suijkerbuijk, B. M. J. M.; Klein Gebbink, R. J. M. Merging Porphyrins with Organometallics: Synthesis and Applications. Angew. Chem. Int. Edit. 2008, 47, 7396-7421.

(2) Shubina, T. E.; Marbach, H.; Flechtner, K.; Kretschmann, A.; Jux, N.; Buchner, F.; Steinrück, H.-P.; Clark, T.; Gottfried, J. M. Principle and Mechanism of Direct Porphyrin Metalation: Joint Experimental and Theoretical Investigation. J. Am. Chem. Soc. 2007, 129, 94769483.

(3) Fuhrhop, J.-H. In Porphyrins and Metalloporphyrins.; Smith, K. M., Ed.; Elsevier Scientific Publishing Company: Amsterdam, 1975; p 593.

(4) Gust, D.; Moore, T. a.; Moore, A. L. Molecular Mimicry of Photosynthetic Energy and Electron Transfer. Acc. Chem. Res. 1993, 26, 198-205.

(5) Kurreck, H.; Huber, M. Model Reactions for Photosynthesis-Photoinduced Charge and Energy Transfer between Covalently Linked Porphyrin and Quinone Units. Angew. Chem. Int. Edit. 1995, 34, 849-866.

(6) Hsiao, J.-S.; Krueger, B. P.; Wagner, R. W.; Johnson, T. E.; Delaney, J. K.; Mauzerall, D. C.; Fleming, G. R.; Lindsey, J. S.; Bocian, D. F.; Donohoe, R. J. Soluble Synthetic Multipor- 
phyrin Arrays. 2. Photodynamics of Energy-Transfer Processes. J. Am. Chem. Soc. 1996, $118,11181-11193$.

(7) Jensen, K. K.; van Berlekom, S. B.; Kajanus, J.; Mårtensson, J.; Albinsson, B. Mediated Energy Transfer in Covalently Linked Porphyrin Dimers. J. Phys. Chem. A 1997, 101, 22182220 .

(8) Pettersson, K.; Kyrychenko, A.; Rönnow, E.; Ljungdahl, T.; Mårtensson, J.; Albinsson, B. Singlet Energy Transfer in Porphyrin-based Donor-Bridge-Acceptor Systems: Interaction between Bridge Length and Bridge Energy. J. Phys. Chem. A 2006, 110, 310-318.

(9) Strachan, J.-P.; Gentemann, S.; Seth, J.; Kalsbeck, W. A.; Lindsey, J. S.; Holten, D.; Bocian, D. F. Effects of Orbital Ordering on Electronic Communication in Multiporphyrin Arrays. J. Am. Chem. Soc. 1997, 119, 11191-11201.

(10) Förster, T. Zwischenmolekulare Energiewanderung und Fluoreszenz. Ann. Phys. 1948, 437, 55-75.

(11) Dexter, D. L. A Theory of Sensitized Luminescence in Solids. J. Chem. Phys. 1953, 21, 836-850.

(12) Montes, V. A.; Pérez-Bolívar, C.; Agarwal, N.; Shinar, J.; Anzenbacher, P. Molecular-wire Behavior of OLED Materials: Exciton Dynamics In Multichromophoric Alq3-oligofluorenePt(II)porphyrin Triads. J. Am. Chem. Soc. 2006, 128, 12436-12438.

(13) Rai, S.; Ravikanth, M. Singlet-singlet Energy Transfer in Homo- and Hetero-Porphyrin Dyads Containing Meso-Tolylporphyrin and Meso-Furylporphyrin Sub-units. Chem. Phys. Lett. 2008, 453, 250-255.

(14) Song, H.-E.; Taniguchi, M.; Speckbacher, M.; Yu, L.; Bocian, D. F.; Lindsey, J. S.; Holten, D. Excited-state Energy Flow in Phenylene-linked Multiporphyrin Arrays. J. Phys. Chem. B 2009, 113, 8011-8019. 
(15) Curutchet, C.; Mennucci, B.; Scholes, G. D.; Beljonne, D. Does Förster Theory Predict the Rate of Electronic Energy Transfer for a Model Dyad at Low Temperature? J. Phys. Chem. B 2008, 112, 3759-3766.

(16) Fückel, B.; Köhn, A.; Harding, M. E.; Diezemann, G.; Hinze, G.; Basché, T.; Gauss, J. Theoretical Investigation of Electronic Excitation Energy Transfer in Bichromophoric Assemblies. J. Chem. Phys. 2008, 128, 074505-1-074505-13.

(17) Chen, H.-C.; You, Z.-Q.; Hsu, C.-P. The Mediated Excitation Energy Transfer: Effects of Bridge Polarizability. J. Chem. Phys. 2008, 129, 084708-1-084708-10.

(18) Curutchet, C.; Feist, F. A.; Van Averbeke, B.; Mennucci, B.; Jacob, J.; Müllen, K.; Basché, T.; Beljonne, D. Superexchange-mediated Electronic Energy Transfer in a Model Dyad. Phys. Chem. Chem. Phys. 2010, 12, 7378-7385.

(19) Caprasecca, S.; Curutchet, C.; Mennucci, B. Toward a Unified Modeling of Environment and Bridge-Mediated Contributions to Electronic Energy Transfer: A Fully Polarizable QM/MM/PCM Approach. J. Chem. Theory Comput. 2012, 8, 4462-4473.

(20) Tomasi, J.; Mennucci, B.; Cammi, R. Quantum Mechanical Continuum Solvation Models. Chem. Rev. 2005, 105, 2999-3093.

(21) Curutchet, C.; Muñoz Losa, A.; Monti, S.; Kongsted, J.; Scholes, G. D.; Mennucci, B. Electronic Energy Transfer in Condensed Phase Studied by a Polarizable QM/MM Model. J. Chem. Theory Comput. 2009, 5, 1838-1848.

(22) Cancès, E.; Mennucci, B.; Tomasi, J. A New Integral Equation Formalism for the Polarizable Continuum Model: Theoretical Background and Applications to Isotropic and Anisotropic Dielectrics. J. Chem. Phys. 1997, 107, 3032-3041.

(23) Cammi, R.; Mennucci, B. Linear Response Theory for the Polarizable Continuum Model. J. Chem. Phys. 1999, 110, 9877-9886. 
(24) Iozzi, M. F.; Mennucci, B.; Tomasi, J.; Cammi, R. Excitation Energy Transfer (EET) between Molecules in Condensed Matter: a Novel Application of the Polarizable Continuum Model (PCM). J. Chem. Phys. 2004, 120, 7029-7040.

(25) Frisch, M. J. et al. Gaussian 09 Revision A.1. 2009; www . gaussian . com, Gaussian, Inc., Wallingford CT.

(26) Sing, U. C.; Kollman, P. An Approach to Computing Electrostatic Charges for Molecules. J. Comput. Chem. 1984, 5, 129-145.

(27) Besler, B. H.; Merz Jr, K. M.; Kollman, P. A. Atomic Charges Derived from Semiempirical Methods. J. Comput. Chem. 1990, 11, 431-439.

(28) Thole, B. T. Molecular Polarizabilities Calculated with a Modified Dipole Interaction. Chem. Phys. 1981, 59, 341-350.

(29) van Duijnen, P. T.; Swart, M. Molecular and Atomic Polarizabilities: Thole's Model Revisited. J. Phys. Chem. A 1998, 102, 2399-2407.

(30) Senn, H. M.; Thiel, W. QM/MM Methods for Biomolecular Systems. Angew. Chem. Int. Ed. 2009, 48, 1198-229.

(31) Seth, J.; Palaniappan, V.; Wagner, R. W.; Johnson, T. E.; Lindsey, J. S.; Bocian, D. F. Soluble Synthetic Multiporphyrin Arrays. 3. Static Spectroscopic and Electrochemical Probes of Electronic Communication. J. Am. Chem. Soc. 1996, 11194-11207.

(32) Albinsson, B.; Mårtensson, J. Long-range Electron and Excitation Energy Transfer in DonorBridge-Acceptor Systems. J Photoch. Photobio. C 2008, 9, 138-155. 